\title{
The molecular basis of anaphase $A$ in animal cells
}

\author{
Uttama Rath • David J. Sharp
}

Published online: 2 April 2011

(C) Springer Science+Business Media B.V. 2011

\begin{abstract}
The mechanisms that move chromatids poleward during anaphase A have fascinated researchers for decades. There is now growing evidence that this movement is tightly linked to the active depolymerization of both ends of kinetochoreassociated microtubules, a mechanism we refer to as "Pacman-Flux." Contemporary data suggest that this is catalyzed by the integration of multiple enzymatic activities including (1) microtubule-end depolymerases housed at the pole or kinetochore, (2) microtubule-severing enzymes used to uncap the ends of kinetochore-associated microtubules, and (3) molecular motors which drive tubulins towards the pole or into kinetochores.
\end{abstract}

Keywords Mitosis $\cdot$ Spindle $\cdot$ Anaphase A · Pacman . Poleward Flux

Abbreviations

Asp Abnormal spindle protein

Gamma-TURC Gamma-tubulin ring complex

Kat-60 Katanin catalytic subunit p60

Kif2A Kinesin heavy chain member 2A

Responsible Editors: James Wakefield and Herbert Macgregor

U. Rath $\cdot$ D. J. Sharp $(\bowtie)$

Department of Physiology and Biophysics,

Albert Einstein College of Medicine,

1300 Morris Park Avenue,

Bronx, NY 10461, USA

e-mail: david.sharp@einstein.yu.edu
Kif2B

Kif2C

KLP10A

KLP59C

KLP59D

KLP61F

NEB

NuMA

ZW10
Kinesin heavy chain member $2 \mathrm{~B}$

Kinesin heavy chain member $2 \mathrm{C}$

Kinesin-like protein $10 \mathrm{~A}$

Kinesin-like protein 59C

Kinesin-like protein 59D

Kinesin-like protein $61 \mathrm{~F}$

Nuclear envelope breakdown

Nuclear mitotic apparatus protein

Zeste White 10

The segregation of chromosomes into the daughter cell products of cell division is achieved during mitotic anaphase, when sister chromatids disjoin and are transported to their respective destinations by the microtubule-based spindle apparatus. The longitudinal splitting of chromosomes was most famously illustrated by the nineteenth-century German Anatomist, Walther Flemming, who named the entire process "mitosis" (from the Greek word mitos meaning thread) based on the thread-like appearance of chromosomes (Flemming 1882). Decades of advancements in microscopy and the development of approaches for manipulating protein function in cells have led to increasingly complex insights into both the phenomenological and molecular underpinnings of mitosis. We are now in a position to speculate with some authority about the specific molecular pathways that underlie spindle assembly, chromosome motility, and segregation. Here, we focus on our emerging understanding of the molecular basis of perhaps the 
most dramatic of all mitotic events, the disjunction and poleward movement of sister chromatids during anaphase A.

Anaphase must be understood within the broader context of the entire process of mitosis. The earliest phase of mitosis, known as prophase, is marked by the condensation of duplicated chromosomes which occurs prior to nuclear envelope breakdown (NEB). NEB stimulates the onset of prometaphase, when chromosomes are captured by spindle microtubules, many of which are nucleated from duplicated centrosomes, and maneuvered to the spindle equator or metaphase plate. Metaphase begins once all chromosomes have achieved an equatorial position. During this time, chromosomes often continue to oscillate back and forth across the metaphase plate (Skibbens et al. 1993). The metaphase-to-anaphase transition is then signaled by a dramatic shift in cell chemistry resulting in the disjunction and poleward separation of sister chromatids. This occurs in two subphases: anaphase A chromatid-to-pole motion and anaphase B spindle elongation. The separated chromosomal masses finally decondense into daughter nuclei during telophase.

How does the spindle move chromosomes? The primary functional interface between chromosomes and spindle microtubules is the kinetochore, a multiprotein complex that assembles onto the centromere of each sister chromatid. Early in prometaphase, kinetochores can attach to spindle microtubules laterally leading to their poleward transport by the minus-end directed motor cytoplasmic dynein (Pfarr et al. 1990; Steuer et al. 1990; Wordeman et al. 1991). However, as mitosis progresses, kinetochores capture and make stable associations with numerous microtubule plus-ends (Cheeseman and Desai 2008), and it is in this configuration that the majority of chromosome movements, including anaphase, occur.

Over the years, two main models have emerged to describe the role of kinetochores and their associated microtubules in the poleward translocation of chromosomes during anaphase. The first posited that kinetochore microtubules serve as traction fibers which pull kinetochores (and chromatids) poleward (Schrader 1953; Pickett-Heaps et al. 1997). This evolved into the "Flux" model based on observations that tubulin subunits within kinetochore-microtubules Flux poleward through most of mitosis (Rogers et al. 2005). "Flux" is now known to be promoted by the depolymerization of spindle pole-focused microtubule minus-ends (Rogers et al. 2005). Linkage of kinetochores to fluxing tubulins would provide a means of reeling chromatids into the poles. A second model, known as "Pacman" (after the video game), suggested that factors within kinetochores actively depolymerize kinetochore-associated microtubule plus-ends, thereby allowing chromatids to "chew" their way poleward along microtubule tracks (Pickett-Heaps et al. 1982). While there has been some controversy as to whether one or the other mechanism is the most important factor in anaphase A, it is now known that both play a role and often occur simultaneously (but to various extents depending on cell type). Thus, we refer to the general mechanism of anaphase A as "Pacman-Flux." A survey of current work also suggests the existence of a fantastic symmetry in the molecular basis of Pacman and Flux-based anaphase A with both emerging from the active depolymerization of kinetochore microtubule ends coupled to motor-driven microtubule sliding (Fig. 1A).

\section{Reeling chromatids into poles by poleward Flux}

The first published description of Fluxing spindle fibers (microtubules) can be attributed to Arthur Forer in the 1960s (Forer 1965). Using a UV microbeam to irradiate the spindle fibers of crane fly spermatocytes, Forer noticed that the resulting region of reduced birefringence moved toward the poles and then disappeared. Forer also noted that the rate of this Flux approximated that of anaphase A. Based on these observations, he concluded that "the area of reduced birefringence is just a marker in this continuously moving system" and that spindle fiber components move toward the pole where they are disassembled and recycled (Forer 1965).

This idea was developed further by Margolis and Wilson who posited that spindle microtubules function as molecular treadmills based on observations of microtubule treadmilling in vitro (Margolis and Wilson 1978). In particular, Margolis and Wilson speculated that tightly controlled modifications of such behaviors within the spindle could be used to control the position of chromosomes. During metaphase, plus-end polymerization at kinetochores balanced by minus-end depolymerization at the poles would create a steady state in which kinetochore 


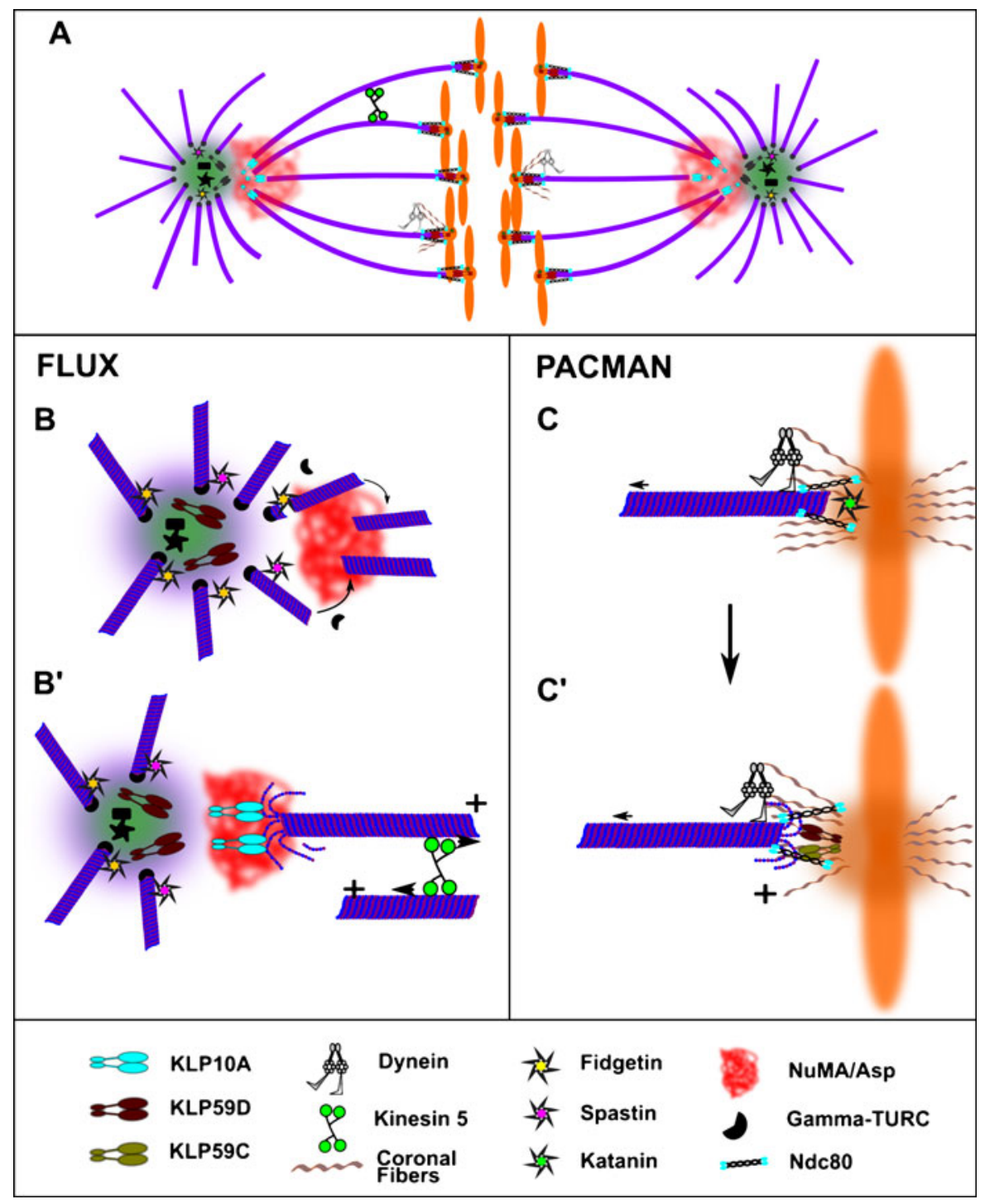

Fig. 1 A model for Pacman-Flux mechanism-based chromosome segregation during anaphase. A Chromosome segregation during anaphase is driven by simultaneous depolymerization of the plus- and the minus-ends of the kinetochore microtubule (kMT) by the "Pacman-Flux" mechanism, a complex process resulting from the culmination of a diverse array of enzymatic activities. $B$ Microtubule minus-ends are released from the $\gamma$-TURC caps at the centrosome by the microtubule-severing enzymes Spastin/Fidgetin. The free minus-ends are captured by the insoluble spindle pole matrix $(N u M A / A s p)$. $B^{\prime}$ Kinesin-13s tethered to the spindle pole matrix $(K L P 10 A)$ depolymerize the free minus-ends of the kMT, thereby driving poleward Flux and reeling in the attached chromosomes. Additionally, microtubule sliding by homotetrameric kinesin 5 (KLP61F/Eg5) contributes

microtubules maintain a constant length, albeit with the tubulin subunits within them flowing poleward, to Flux by pushing microtubules toward the poles. $C$ Microtubule plus-ends embedded in the kinetochore are uncapped by microtubule-severing enzyme, Katanin. The uncapped microtubule end is linked to the kinetochore via multiple interactions of $\mathrm{Ndc80}$. Dynein attached to the coronal fibers and the microtubule also keeps the plus-ends tethered to the kinetochore. $C^{\prime}$ Kinesin-13s, KLP59D or KLP59C (In Drosophila S2 cells and embryos, respectively), depolymerize the uncapped plus-ends to drive Pacman motility-based chromosome segregation. Dynein attached to the coronal fibers walks toward the minus ends of the kinetochore microtubules, thereby pushing the microtubule into the depolymerizing machinery at the kinetochores

allowing chromosomes to persist at the spindle equator (Margolis and Wilson 1981; Margolis and 
Wilson 1978). The cessation of plus-end polymerization at the onset of anaphase would cause kinetochoreassociated microtubules to shorten, through unbalanced minus-end depolymerization, thus reeling chromatids into the pole.

Direct observation of the Flux of tubulin subunits within spindle microtubules had to wait until the mid1980s. This was first demonstrated by Mitchison and colleagues who injected biotin-labeled tubulins into live monkey fibroblast (BSC1) and followed their incorporation into kinetochore microtubules using electron microscopy. New tubulin subunits were observed to incorporate primarily at the plus ends and then spread poleward over time (Mitchison et al. 1986). Similar results were obtained when the spindles of LL-CPK1 and PtK2 cells were labeled with photoactivatable caged fluorophore-tagged tubulins. Live observation of photoactivated bands within the half spindles of these cells revealed the continual poleward flow of the tagged tubulin subunits (Mitchison et al. 1986; Mitchison 1989; Gorbsky et al. 1987; Gorbsky et al. 1988).

It was not until the last decade that the first molecular components of the Flux "machinery" were identified. And as additional components continue to be found, it appears that Flux within anaphase spindles generally involves at least three distinct enzymatic activities: (1) a pole-associated minus-end depolymerase, (2) centrosome-associated microtubulesevering enzymes, and (3) microtubule-sliding motors within the central spindle (Fig. 1B, B').

Mechanism of Flux I: Depolymerization of spindle pole-focused microtubule minus-ends by kinesin-13s

Probably the best known and characterized of the protein factors involved in the stimulation of Flux are the kinesin-13s. Members of this kinesin subfamily are immotile but instead bind to microtubule ends and catalyze their depolymerization, in vitro (Desai et al. 1999). Work in a variety of systems, ranging from fruitflies to humans, has indicated that kinesin-13s, positioned on spindle poles, promote Flux by depolymerizing pole-focused minus-ends.

The first kinesin-13 with a definitively identified role in the generation of Flux-based anaphase A was Drosophila KLP10A. Although Drosophila contains two additional kinesin-13 family members (both of which are discussed below), KLP10A is the only one to associate with spindle poles (note that the spindle pole is a clearly distinct entity from the centrosome in Drosophila cells; (Rogers et al. 2005)). KLP10A's mitotic function was initially demonstrated in syncytial blastoderm-stage embryos. Specifically, injection of embryos with anti-KLP10A antibodies was found to induce the near complete cessation of poleward Flux in both metaphase and anaphase spindles and a commensurate decrease in the rate of anaphase A (Rogers et al. 2004). In embryos, Flux occurs at roughly $40 \%$ of the total anaphase A rate. Depletion of KLP10A from cultured S2 cells using doublestranded RNA-mediated interference (RNAi) was later shown to have a similar effect (Buster et al. 2007; Rath et al. 2009). Inhibition of pole-associated kinesin-13s in vertebrate spindles has been found to reduce Flux rate and anaphase $\mathrm{A}$ indicating that this portion of the Flux machinery is highly conserved (Ganem et al. 2005).

The mechanisms used to target kinesin-13s to spindle poles have also received attention. Studies in vertebrates and fruitflies have suggested that these proteins are transported poleward by cytoplasmic dynein. Inhibition of dynein in Xenopus egg extract spindles or Drosophila tissue culture cells causes kinesin-13s that are normally associated with poles to become spread across the spindle (Gaetz and Kapoor 2004; Morales-Mulia and Scholey 2005). We have also found that the accumulation of KLP10A on spindle poles involves a second kinesin-13 family member, KLP59D. Interestingly, KLP59D appears to work by shuttling KLP10A through centrosomes (KLP59D localizes to centrosomes but not poles) and we have proposed that this generates a localized region of active depolymerase activity in the vicinity of the pole (Rath et al. 2009). Within the cytoplasm, the majority of KLP10A proteins are known to be maintained in a low activity state, through the phosphorylation of their motor domains (Mennella et al. 2009). Passage through the centrosome, which houses numerous phosphatases (as well as kinases), could provide a means of locally activating a subpopulation of the protein. Finally, we have found that the pole association of KLP10A is entirely dependent on the protein Asp (Saunders et al. 1997; Wakefield et al. 2001), a highly coiled-coil protein thought to form an insoluble pole matrix (unpublished data). The vertebrate protein NuMA has also been proposed to form such a pole matrix (Dionne et al. 
1999). Asp/NuMA could work to immobilize kinesin$13 \mathrm{~s}$ released from the centrosome or transported poleward by cytoplasmic dynein. Of course, this awaits further investigation.

Mechanism of Flux part II: Creation of free microtubule minus-ends by microtubule-severing enzymes

Kinesin-13s are not the only proteins involved in promoting the depolymerization of microtubule minus-ends at the poles. Recent work in Drosophila has shown that this also involves two putative microtubule-severing enzymes-proteins with the capacity to cut the microtubule lattice in vitro (RollMecak and McNally 2010)-housed within the centrosome. As with KLP10A, depletion of either these, termed Dm-Spastin and Dm-Fidgetin, from Drosophila tissue culture cells suppresses Flux and anaphase A. Moreover, fluorescence recovery after photobleaching studies revealed that the loss of either protein strongly suppressed turnover of tubulin subunits at the poles (Zhang et al. 2007). How do centrosomeassociated microtubule-severing proteins carry out such a function? We propose that this occurs as follows: Centrosome-nucleated microtubules are initially capped at their minus-ends by their nucleating gamma-tubulin ring complexes shielding them from the action of KLP10A (Wiese and Zheng 2000; Keating and Borisy 2000). This cap is removed by Dm-Spastin and/or Fidgetin-mediated severing near the centrosome which creates a free minus-end that is incorporated into the pole (perhaps via an association with NuMA/Asp). This end is now a suitable substrate for KLP10A-catalyzed depolymerization. We note that another minus-end capping protein, termed Patronin, has also been recently shown to antagonize KLP10A and serve as a brake on poleward Flux (Goodwin and Vale 2010). Dm-Spastin and Dm-Fidgetin could work by removing Patronin from microtubule minus-ends, as well.

Why Flux involves both Dm-Spastin and Dm-Fidgetin remains unknown. While a similar role for microtubule-severing enzymes in systems other than Drosophila has not been reported in the literature, our preliminary findings indicate that human Fidgetin localizes to centrosomes and promotes poleward Flux (our unpublished observations), suggesting that this portion of the Flux machinery is also evolutionarily conserved.
Mechanism of Flux part III: Kinesin-5's slide microtubules toward the poles

Finally, in addition to MT minus-end depolymerization, Flux involves the poleward sliding of microtubules by kinesin- $5 \mathrm{~s}$ which feed minus-ends into the depolymerase machinery at the poles. Microtubule sliding was initially proposed as a mechanism for anaphase A by McIntosh et al. (McIntosh et al. 1969), and a number of kinesins have now been identified which have the capacity to slide microtubules relative to one another (Sharp et al. 2000c). Among the most widely studied of these are the kinesin- $5 \mathrm{~s}$. These plusend directed motors assemble into bipolar homotetramers with two motor domains positioned at opposite ends of a central rod. This allows them to cross-link and slide apart antiparallel microtubules with their minus-ends leading (Kashina et al. 1996; Sharp et al. 1999; Kapitein et al. 2005; van den Wildenberg et al. 2008).

Owing to their structure and transport properties, Kinesin-5s have been considered as ideal candidates to slide microtubules toward spindle poles and thus contribute to Flux. However, direct demonstration of this was made difficult due to the fact that these enzymes are also required to establish and/or maintain spindle bipolarity - their inhibition usually results in the formation of monopolar spindles (Sharp et al. 2000b). Miyomoto and coworkers were able to circumvent this in Xenopus egg extracts by studying spindles formed after the coinhibition the kinesin-5, Eg5, and cytoplasmic dynein. Under these conditions, bipolar spindles form but display a reduced Flux rate. In a separate set of studies, these authors prevented spindle collapse by gently squashing spindles between two coverslips (Miyamoto et al. 2004). Again, the inhibition of Eg5 (without the coinhibition of dynein) was found to induce a significant decrease in the rate of Flux.

Brust-Mascher et al. used a different approach to show that the Drosophila kinesin-5, KLP61F, is required for normal Flux and anaphase A. By injecting anti-KLP61F antibodies into syncytial embryos (which have a two-dimensional sheet of spindles just beneath their surface), the authors were able to generate a gradient of phenotypes, due to the diffusion of the inhibitor, with spindles collapsing near the injection site while maintaining their bipolarity at more distal sites. The shortened but still bipolar spindles formed 
under these conditions were found to display significantly reduced Flux and anaphase A rates (BrustMascher et al. 2009). The authors have gone on to show that through most of mitosis, KLP61Fmediated microtubule sliding is buffered by the depolymerization of pole-associated minus-ends by KLP10A. However, downregulation of KLP10A at the onset of anaphase B results in a "switch" from Flux to sliding that drives spindle elongation (BrustMascher and Scholey 2002). This model is specifically supported by the observations that (1) Flux ceases at the onset of anaphase B (Brust-Mascher and Scholey 2002) and (2) the inhibition of KLP10A causes metaphase spindles to elongate at anaphase B rates (Rogers et al. 2004).

There is still some question as to how kinesin- $5 \mathrm{~s}$ are able to generate the sliding of microtubules within the half spindles. This is particularly so of kinetochore microtubules which contain primarily parallel microtubules. One possibility is that kinetochore microtubules are cross-linked (either by kinesin-5s or other cross-linkers) and slide relative to nonkinetochore microtubules emerging from the opposite pole (Scholey et al. 2001). Another possibility is that kinesin-5s are fixed on and slide microtubules relative to a static spindle matrix (Kapoor and Mitchison 2001). Of course, these are not mutually exclusive possibilities.

The relative contribution of Flux to anaphase A varies by cell type

While Flux occurs during anaphase in the spindles from evolutionarily diverse cell types and during both meiosis and mitosis, its potential contribution to anaphase A can vary greatly. For example, in crane fly spermatocytes, Flux occurs at nearly twice the rate of anaphase A, since the plus-ends of kinetochore microtubules continue to polymerize even as the kinetochore is pulled poleward (LaFountain et al. 2004). Similarly in Xenopus egg extract spindles, the rates of Flux and anaphase $\mathrm{A}$ are almost identical (Desai et al. 1998). In contrast, in Drosophila, Flux provides a smaller contribution to chromatid-to-pole motion during mitosis. In Drosophila embryos and tissue culture cells, Flux has been measured at $\sim 50 \%$ of anaphase A rates (Rogers et al. 2004; Goshima and Vale 2005; Buster et al. 2007; Zhang et al. 2007; Rath et al. 2009). And in many vertebrate cell types, the potential contribution of Flux to anaphase A decreases to $20-30 \%$ (Ganem et al. 2005).

\section{Pacman-based depolymerization of kinetochore- associated microtubule plus-ends allows chromosomes to chew-up microtubule tracks}

Chromosome movement due to the depolymerization of kinetochore-associated microtubule plus-endstermed Pacman (Cassimeris et al. 1987) - has long been thought to provide a driving force for anaphase A (Pickett-Heaps et al. 1982). The first direct observations of Pacman-based anaphase A came from some of the same studies credited with the identification of Flux. For example, Mitchison et al. (1986) injected BSC1 cells with biotinylated tubulin, fixed the cells at various time points during metaphase and anaphase, and localized the modified tubulin within the spindle. As mentioned above, in metaphase spindles, the labeled tubulin was present in MTs near the kinetochores, suggesting assembly primarily at the plus-ends. In contrast, during anaphase, about $60 \%$ of the microtubules no longer contained modified tubulin, leading to the conclusion that the labeled regions of microtubules near the kinetochores had been disassembled (Mitchison et al. 1986). However, the most definitive early proof of a "Pacman" activity at the kinetochore came from work of Gorbsky and colleagues, who injected LLC-PK1 cells with fluorescent tubulin, and photobleached bars in the half spindles during anaphase. Poleward moving chromatids were observed to approach and pass through the bleach zone strongly supporting the hypothesis that their movement involved the depolymerization of kinetochore-associated microtubule plus-ends (Gorbsky et al. 1987; Gorbsky et al. 1988).

A similar conclusion was drawn from studies in grasshopper spermatocytes using an entirely different experimental paradigm. In these studies, kinetochore microtubules were severed from the pole at the onset of anaphase. Chromatids were found to move toward the minus-ends of these fibers, despite their complete dissociation from the pole (which was thought to house the engine for poleward chromosome movement) leading to the conclusion that chromosome movement is generated, at least in part, by proteins at or near kinetochores which likely induce the depoly- 
merization of kinetochore microtubule plus-ends (Nicklas 1989).

Though work from many groups provided evidence for the existence of force producers at or near the kinetochores, the molecular components involved in Pacman-based anaphase A remained elusive until relatively recently. It now appears that the basic mechanism used to stimulate Pacman is strikingly similar to that of Flux. In particular, Pacman likely involves the integration of (1) a microtubule plus-end depolymerase in the kinetochore interior, (2) severing enzyme to uncap the plus-ends of kinetochore microtubules, (3) a microtubule sliding motor to feed plusends into the kinetochore, and (4) a linker protein allowing kinetochores to remain attached to depolymerizing plus-ends (Fig. 1C, $\mathrm{C}^{\prime}$ ).

Mechanism of Pacman I: Depolymerization of kinetochore-associated microtubule plus-ends by kinesin-13s

As with Flux, which is promoted by kinesin-13s positioned on the poles, efficient Pacman requires the activity of kinesin-13s housed within the kinetochore. However, the specific kinesin-13s used in each process appear to be distinct probably allowing Flux and Pacman to be regulated independently. This has probably been most thoroughly demonstrated in Drosophila, which contains three kinesin-13 family members termed KLP10A, KLP59C, and KLP59D. As mentioned above, KLP10A targets to poles and promotes flux in this system. Alternatively, Pacmanbased anaphase A in syncytial blastoderm-stage embryos is stimulated by KLP59C, which targets specifically to centromeres/inner kinetochores. Inhibition of KLP59C in embryos via antibody microinjection has been found to strongly reduce the rate of anaphase A, $\sim 60 \%$, due solely to the cessation of Pacman (Rogers et al. 2004). Under these conditions, kinetochores become passively anchored to fluxing kinetochore microtubules and are reeled into poles thusly.

Interestingly, Drosophila tissue culture cells do not require KLP59C for anaphase A but instead utilize KLP59D, which is also positioned on kinetochores (Rath et al. 2009). The basis for this is unclear but may be related to KLP59C's unique ability among the Drosophila kinesin-13s to induce plus-end depoly- merization by suppressing rescues (transitions from shrinkage to growth). Elegant modeling studies have suggested that an antirescue factor within the kinetochore is particularly well suited to promote the relatively fast anaphase A rates observed during embryonic mitoses (Civelekoglu-Scholey et al. 2006). Finally, like Drosophila, humans contain three kinesin-13 family members, Kif2A, Kif2B, and $\mathrm{Kif} 2 \mathrm{C}$, and while the situation is complex, there is evidence that Kif2A is utilized to promote Flux while Kif2B drives Pacman (Kif2C corrects errors in kinetochore microtubule attachments.); (Manning et al. 2007; Kline-Smith et al. 2004).

Mechanism of Pacman II: Uncapping of kinetochoreassociated microtubule plus-ends by microtubulesevering enzymes

Pacman, at least in Drosophila, also requires the activity of a microtubule-severing enzyme-the Drosophila katanin, Dm-Kat-60 - yet again suggesting its overwhelming mechanistic similarity to Flux. Unlike the related proteins Dm-Spastin and Dm-Fidgetin which associate with centrosomes, Kat-60 localizes to kinetochores and chromosome arms. The depletion of this protein from tissue culture cells slows anaphase A and does so entirely by suppressing Pacman (Zhang et al. 2007).

We have proposed that Kat-60 stimulates Pacman by uncapping kinetochore-associated microtubule plus-ends. While the need to remove plus-end caps has not been widely considered, polymerizing microtubule plus-ends in cells are known to be coated by plus-end tracking proteins, such as EB1 (MimoriKiyosue et al. 2000). EB1 has been found to promote plus-end polymerization (Ligon et al. 2003), and a related protein, EB3, was recently shown to shield microtubule ends from kinesin-13-induced depolymerization in vitro (Montenegro Gouveia et al. 2010). Severing of microtubules in the region of the kinetochore could provide an efficient mechanism for removing these caps and generating a bare end that can then be attacked by kinesin-13s. Severing along chromosome arms could also be utilized to remove other types of capping proteins. One prime set of candidates is the chromokinesins - kinesins which bind to chromosome arms and link them to microtubules. Some chromokinesins have the capacity to stabilize 
microtubules in vitro, and thus, their removal from the microtubule ends could reduce steric hindrance on poleward moving chromosomes thus aiding in Pacman (Bringmann et al. 2004).

Kat-60 is likely to be maintained under tight regulatory control, as it has no strong influence on chromosome movements prior to anaphase (Zhang et al. 2007). The activation of Kat-60 (perhaps through alterations of its phosphorylation state or degradation of a specific binding partner) could induce the transition of kinetochore-associated microtubule plus-ends from net polymerization to depolymerization thus triggering the onset of anaphase A.

Mechanism of Pacman III: Cytoplasmic dynein feeds kinetochore-associated microtubule plus-ends into the kinetochore

In addition to the plus-end depolymerization machinery, Pacman has been proposed to involve a forcegenerating element at the kinetochore, namely the minus-end directed motor cytoplasmic dynein. Indeed, numerous studies have revealed important roles for dynein in anaphase A. Global inhibition of dynein within Drosophila early embryos was shown to significantly reduce anaphase A to rates approximating those later observed following the inhibition of the Pacman depolymerase in this system, KLP59C (Sharp et al. 2000a; Rogers et al. 2004). Moreover, analyses of chromosome movements in Drosophila spermatocytes carrying mutations in the genes encoding zw10 or rod, two proteins specifically involved in targeting dynein to kinetochores but not elsewhere in the spindle, revealed a similar attenuation of chromatid-to-pole motion (Savoian et al. 2000). Dynein's role in this process is likely evolutionarily conserved as siRNA depletion of human ZW10 from U2OS cells reduces anaphase A by $\sim 40 \%$ (Yang et al. 2007).

How would dynein contribute to Pacman-based anaphase A? Dynein is positioned on the coronal fibers that extend out from the exterior kinetochore face, positioning it appropriately to make substantial lateral contacts with the microtubule lattice (Pfarr et al. 1990; Steuer et al. 1990; Wordeman et al. 1991). We suggest that from this position, dynein can capture and feed microtubule plus-ends into the depolymerization machinery housed within the kinetochore. In this regard, its activity would be somewhat analogous to the related activity of kinesin-5s which slide microtubule minus-ends toward the pole. However, it should be noted that dynein's contribution to Pacman may vary as most if not all of the motor is lost from the kinetochore during anaphase in some cell types (King et al. 2000).

Mechanism of Pacman IV: Linkage of kinetochores to shortening microtubule plus-ends

The final component for Pacman-based anaphase A is a linker between kinetochores and the constantly shortening microtubule plus-ends contained within them. Multiple low affinity linkers have been proposed to keep chromosomes tethered to the ends of dynamic microtubules (Powers et al. 2009). Among these, the $\mathrm{Ndc} 80$ complex is now viewed as the most likely candidate to maintain the attachment between kinetochores and depolymerizing plus-ends during anaphase A. The Ndc80 complex is thought to be the core-binding site between kinetochores and microtubules, and its components are conserved from fungi to the humans. It consists of four proteins (Hec1, Nuf2, Spc25, and Spc24) arranged as a long coiledcoil rod with globular heads at either end. Nuf2/Hec1 domains attach to microtubule ends at the outer kinetochore while the Spc24/25 proteins are embedded in the inner kinetochore (Ciferri et al. 2007; Joglekar et al. 2010). Ndc80 complexes could serve as "fingers" which rapidly attach and detach from individual tubulins within the microtubule lattice.

\section{Conclusion}

The molecular machinery used to move separated sister chromatids towards opposite spindle poles is complex, involving a diverse array of distinct enzymatic activities. Yet, it is also elegant in the integration of these. This movement is linked to the depolymerization of both ends of kinetochoreassociated microtubules, a mechanism we refer to as Pacman-Flux. And while different proteins are used to catalyze the depolymerization of each end, the basic mechanism is apparently quite similar involving (1) a microtubule-destabilizing kinesin-13 positioned at the pole or in the kinetochore, (2) a microtubule-severing enzyme to prepare each end for kinesin-13-induced depolymerization, and (3) a molecular motor to help power movement and feed microtubule ends into the 
depolymerase machinery. Linker proteins that attach kinetochores and poles to depolymerizing microtubule ends are also likely components of this "PacmanFlux machinery."

Of course, what we have presented here is only part of the picture. Numerous additional components required for efficient anaphase A are likely to emerge, and those identified in certain cell types may play no role in others. There is also evidence that chromatidto-pole motility is not solely the result of the depolymerization of kinetochore-associated microtubule ends (Fabian and Forer 2005; Fabian and Forer 2007). Finally, some cell types display very little anaphase $\mathrm{A}$, and in these anaphase, chromosome segregation is driven almost entirely by anaphase B (Straight et al. 1997). However, we hope to have provided a useful framework for understanding this fundamental and visually striking biological event.

\section{References}

Bringmann H, Skiniotis G, Spilker A, Kandels-Lewis S, Vernos I, Surrey T (2004) A kinesin-like motor inhibits microtubule dynamic instability. Science 303:1519-1522

Brust-Mascher I, Scholey JM (2002) Microtubule flux and sliding in mitotic spindles of Drosophila embryos. Mol Biol Cell 13:3967-3975

Brust-Mascher I, Sommi P, Cheerambathur DK, Scholey JM (2009) Kinesin-5-dependent poleward flux and spindle length control in Drosophila embryo mitosis. Mol Biol Cell 20:1749-1762

Buster DW, Zhang D, Sharp DJ (2007) Poleward tubulin flux in spindles: regulation and function in mitotic cells. Mol Biol Cell 18:3094-3104

Cassimeris LU, Walker RA, Pryer NK, Salmon ED (1987) Dynamic instability of microtubules. Bioessays 7:149-154

Cheeseman IM, Desai A (2008) Molecular architecture of the kinetochore-microtubule interface. Nat Rev Mol Cell Biol 9:33-46

Ciferri C, Musacchio A, Petrovic A (2007) The Ndc80 complex: hub of kinetochore activity. FEBS Lett 581:2862-2869

Civelekoglu-Scholey G, Sharp DJ, Mogilner A, Scholey JM (2006) Model of chromosome motility in Drosophila embryos: adaptation of a general mechanism for rapid mitosis. Biophys J 90:3966-3982

Desai A, Maddox PS, Mitchison TJ, Salmon ED (1998) Anaphase A chromosome movement and poleward spindle microtubule flux occur at similar rates in Xenopus extract spindles. J Cell Biol 141:703-713

Desai A, Verma S, Mitchison TJ, Walczak CE (1999) Kin I kinesins are microtubule-destabilizing enzymes. Cell 96:69-78

Dionne MA, Howard L, Compton DA (1999) NuMA is a component of an insoluble matrix at mitotic spindle poles. Cell Motil Cytoskeleton 42:189-203
Fabian L, Forer A (2005) Redundant mechanisms for anaphase chromosome movements: crane-fly spermatocyte spindles normally use actin filaments but also can function without them. Protoplasma 225:169-184

Fabian L, Forer A (2007) Possible roles of actin and myosin during anaphase chromosome movements in locust spermatocytes. Protoplasma 231:201-213

Flemming, W. 1882. Zellsubstanz, Kern und Zelltheilung ... Mit ... Tafeln, pp. viii. 424. Leipzig

Forer A (1965) Local reduction of spindle fiber birefringence in living nephrotoma suturalis (Loew) spermatocytes induced by ultraviolet microbeam irradiation. J Cell Biol 25 (SUPPL):95-117

Gaetz J, Kapoor TM (2004) Dynein/dynactin regulate metaphase spindle length by targeting depolymerizing activities to spindle poles. J Cell Biol 166:465-471

Ganem NJ, Upton K, Compton DA (2005) Efficient mitosis in human cells lacking poleward microtubule flux. Curr Biol 15:1827-1832

Goodwin SS, Vale RD (2010) Patronin regulates the microtubule network by protecting microtubule minus ends. Cell 143:263-274

Gorbsky GJ, Sammak PJ, Borisy GG (1987) Chromosomes move poleward in anaphase along stationary microtubules that coordinately disassemble from their kinetochore ends. J Cell Biol 104:9-18

Gorbsky GJ, Sammak PJ, Borisy GG (1988) Microtubule dynamics and chromosome motion visualized in living anaphase cells. J Cell Biol 106:1185-1192

Goshima G, Vale RD (2005) Cell cycle-dependent dynamics and regulation of mitotic kinesins in Drosophila S2 cells. Mol Biol Cell 16:3896-3907

Joglekar AP, Bloom KS, Salmon ED (2010) Mechanisms of force generation by end-on kinetochore-microtubule attachments. Curr Opin Cell Biol 22:57-67

Kapitein LC, Peterman EJ, Kwok BH, Kim JH, Kapoor TM, Schmidt CF (2005) The bipolar mitotic kinesin Eg5 moves on both microtubules that it crosslinks. Nature 435:114 118

Kapoor TM, Mitchison TJ (2001) Eg5 is static in bipolar spindles relative to tubulin: evidence for a static spindle matrix. J Cell Biol 154:1125-1133

Kashina AS, Scholey JM, Leszyk JD, Saxton WM (1996) An essential bipolar mitotic motor. Nature 384:225

Keating TJ, Borisy GG (2000) Immunostructural evidence for the template mechanism of microtubule nucleation. Nat Cell Biol 2:352-357

King JM, Hays TS, Nicklas RB (2000) Dynein is a transient kinetochore component whose binding is regulated by microtubule attachment, not tension. J Cell Biol 151:739748

Kline-Smith SL, Khodjakov A, Hergert P, Walczak CE (2004) Depletion of centromeric MCAK leads to chromosome congression and segregation defects due to improper kinetochore attachments. Mol Biol Cell 15:1146-1159

Lafountain JR Jr, Cohan CS, Siegel AJ, Lafountain DJ (2004) Direct visualization of microtubule flux during metaphase and anaphase in crane-fly spermatocytes. Mol Biol Cell 15:5724-5732

Ligon LA, Shelly SS, Tokito M, Holzbaur EL (2003) The microtubule plus-end proteins EB1 and dynactin have 
differential effects on microtubule polymerization. Mol Biol Cell 14:1405-1417

Manning AL, Ganem NJ, Bakhoum SF, Wagenbach M, Wordeman L, Compton DA (2007) The kinesin-13 proteins Kif2a, Kif2b, and Kif2c/MCAK have distinct roles during mitosis in human cells. Mol Biol Cell 18:2970-2979

Margolis RL, Wilson L (1978) Opposite end assembly and disassembly of microtubules at steady state in vitro. Cell $13: 1-8$

Margolis RL, Wilson L (1981) Microtubule treadmills-possible molecular machinery. Nature 293:705-711

Mcintosh JR, Hepler PK, Wie DGV (1969) Model for Mitosis. Nature 224:659-663

Mennella V, Tan DY, Buster DW, Asenjo AB, Rath U, Ma A, Sosa HJ, Sharp DJ (2009) Motor domain phosphorylation and regulation of the Drosophila kinesin 13, KLP10A. J Cell Biol 186:481-490

Mimori-Kiyosue Y, Shiina N, Tsukita S (2000) Adenomatous polyposis coli (APC) protein moves along microtubules and concentrates at their growing ends in epithelial cells. $\mathrm{J}$ Cell Biol 148:505-518

Mitchison TJ (1989) Polewards microtubule flux in the mitotic spindle: evidence from photoactivation of fluorescence. $\mathrm{J}$ Cell Biol 109:637-652

Mitchison T, Evans L, Schulze E, Kirschner M (1986) Sites of microtubule assembly and disassembly in the mitotic spindle. Cell 45:515-527

Miyamoto DT, Perlman ZE, Burbank KS, Groen AC, Mitchison TJ (2004) The kinesin Eg5 drives poleward microtubule flux in Xenopus laevis egg extract spindles. J Cell Biol 167:813-818

Montenegro Gouveia S, Leslie K, Kapitein LC, Buey RM, Grigoriev I, Wagenbach M, Smal I, Meijering E, Hoogenraad CC, Wordeman L, Steinmetz MO, Akhmanova A (2010) In vitro reconstitution of the functional interplay between MCAK and EB3 at microtubule plus ends. Curr Biol 20:1717-1722

Morales-Mulia S, Scholey JM (2005) Spindle pole organization in Drosophila $\mathrm{S} 2$ cells by dynein, abnormal spindle protein (Asp), and KLP10A. Mol Biol Cell 16:3176-3186

Nicklas RB (1989) The motor for poleward chromosome movement in anaphase is in or near the kinetochore. $\mathrm{J}$ Cell Biol 109:2245-2255

Pfarr CM, Coue M, Grissom PM, Hays TS, Porter ME, Mcintosh JR (1990) Cytoplasmic dynein is localized to kinetochores during mitosis. Nature 345:263-265

Pickett-Heaps JD, Tippit DH, Porter KR (1982) Rethinking mitosis. Cell 29:729-744

Pickett-Heaps JD, Forer A, Spurck T (1997) Traction fibre: toward a "tensegral" model of the spindle. Cell Motil Cytoskeleton 37:1-6

Powers AF, Franck AD, Gestaut DR, Cooper J, Gracyzk B, Wei RR, Wordeman L, Davis TN, Asbury CL (2009) The Ndc80 kinetochore complex forms load-bearing attachments to dynamic microtubule tips via biased diffusion. Cell 136:865-875

Rath U, Rogers GC, Tan D, Gomez-Ferreria MA, Buster DW, Sosa HJ, Sharp DJ (2009) The Drosophila kinesin-13, KLP59D, impacts Pacman- and Flux-based chromosome movement. Mol Biol Cell 20:4696-4705

Rogers GC, Rogers SL, Schwimmer TA, Ems-Mcclung SC, Walczak CE, Vale RD, Scholey JM, Sharp DJ (2004) Two mitotic kinesins cooperate to drive sister chromatid separation during anaphase. Nature 427:364-370

Rogers GC, Rogers SL, Sharp DJ (2005) Spindle microtubules in flux. J Cell Sci 118:1105-1116

Roll-Mecak A, Mcnally FJ (2010) Microtubule-severing enzymes. Curr Opin Cell Biol 22:96-103

Saunders RD, Avides MC, Howard T, Gonzalez C, Glover DM (1997) The Drosophila gene abnormal spindle encodes a novel microtubule-associated protein that associates with the polar regions of the mitotic spindle. J Cell Biol 137:881-890

Savoian MS, Goldberg ML, Rieder CL (2000) The rate of poleward chromosome motion is attenuated in Drosophila zw10 and rod mutants. Nat Cell Biol 2:948-952

Scholey JM, Rogers GC, Sharp DJ (2001) Mitosis, microtubules, and the matrix. J Cell Biol 154:261-266

Schrader FPOZCU (1953). Mitosis ... Second edition, pp. xii. 170. Columbia University Press: New York

Sharp DJ, Mcdonald KL, Brown HM, Matthies HJ, Walczak C, Vale RD, Mitchison TJ, Scholey JM (1999) The bipolar kinesin, KLP61F, cross-links microtubules within interpolar microtubule bundles of Drosophila embryonic mitotic spindles. J Cell Biol 144:125-138

Sharp DJ, Rogers GC, Scholey JM (2000a) Cytoplasmic dynein is required for poleward chromosome movement during mitosis in Drosophila embryos. Nat Cell Biol 2:922-930

Sharp DJ, Rogers GC, Scholey JM (2000b) Microtubule motors in mitosis. Nature 407:41-47

Sharp DJ, Rogers GC, Scholey JM (2000c) Roles of motor proteins in building microtubule-based structures: a basic principle of cellular design. Biochim Biophys Acta 1496:128-141

Skibbens RV, Skeen VP, Salmon ED (1993) Directional instability of kinetochore motility during chromosome congression and segregation in mitotic newt lung cells: a push-pull mechanism. J Cell Biol 122:859-875

Steuer ER, Wordeman L, Schroer TA, Sheetz MP (1990) Localization of cytoplasmic dynein to mitotic spindles and kinetochores. Nature 345:266-268

Straight AF, Marshall WF, Sedat JW, Murray AW (1997) Mitosis in living budding yeast: anaphase A but no metaphase plate. Science 277:574-578

Van Den Wildenberg SM, TAO L, Kapitein LC, Schmidt CF, Scholey JM, Peterman EJ (2008) The homotetrameric kinesin-5 KLP61F preferentially crosslinks microtubules into antiparallel orientations. Curr Biol 18:1860-1864

Wakefield JG, Bonaccorsi S, Gatti M (2001) The drosophila protein asp is involved in microtubule organization during spindle formation and cytokinesis. J Cell Biol 153:637-648

Wiese C, Zheng Y (2000) A new function for the gammatubulin ring complex as a microtubule minus-end cap. Nat Cell Biol 2:358-364

Wordeman L, Steuer ER, Sheetz MP, Mitchison T (1991) Chemical subdomains within the kinetochore domain of isolated $\mathrm{CHO}$ mitotic chromosomes. J Cell Biol 114:285-294

Yang Z, Tulu US, Wadsworth P, Rieder CL (2007) Kinetochore dynein is required for chromosome motion and congression independent of the spindle checkpoint. Curr Biol 17:973-980

Zhang D, Rogers GC, Buster DW, Sharp DJ (2007) Three microtubule severing enzymes contribute to the "Pacmanflux" machinery that moves chromosomes. J Cell Biol 177:231-242 